\title{
III.
}

\section{Ueber Centrosomen und Sphären in menschlichen Vorderhornzellen.}

\author{
Von \\ Dr. Rud. Kolster, \\ Docent für pathologische Anatomie.
}

(Mit Tafel II.)

Vor Kurzem habe ich eine längere Arbeit ${ }^{1}$ ) über „die Centralgebilde in Vorderhornzellen der Wirbelthiere" veröffentlicht. Die dort behandelten Thiere waren: Cottus scorpius und quadricornis, Rana temporaria, Tropidonotus natrix, Anguis fragilis, Testudo graeca, Columbo domestica, Oris aries und Bos taurus. Auch über die gleichen Gebilde beim Menschen wurden Angaben, welche ich aber erst später Gelegenheit gefunden habe zu vervollständigen, gegeben.

Soviel ich weiss, sind dieses die ersten Angaben über das Vorkommen dieser wichtigen Gebilde in den entsprechenden Ganglienzellen, aber auch sonst ist das Vorkommen derselben in Nervenzellen nur selten festgestellt worden. Da ich dort die hierhergehörende Literatur berücksichtigt habe, will ich hier nur mittheilen, dass mir seit Abschluss der erwähnten Arbeit noch ein neuer Fundort bekannt geworden ist. Studniěkąa hat in den Ganglienzellen der Gehirnnerven ganz kleiner, etwa $3 \mathrm{~cm}$ langer Exemplare von Lophius diese Structuren ebenfalls gesehen.

Dass dieselben in Nervenzellen bisher so selten gefunden worden sind, ist im Ganzen leicht erklärlich, denn wenige Fälle ausgenommen, versagt die zum Nachweis derselben geeignetste Methode hier gänzlich. Dieses nicht aus dem Grunde, dass dieselbe die Centrosomen nicht färbte, im Gegentheil färbt leider die Heidenhain'sche BordeauxEisenhämatoxylinmethode ausser den Centrosomen noch andere Bestandtheile der Ganglienzellen, wie z. B. Pigment und Nissl'sche

1) Anatomische Hefte. Heft 50.

2) Studnička, Beiträge zur Kenntniss der Ganglienzellen. 1. Ein neuer Befund von Centrosomen. Sitzber. d. k. böhm. Gesellschaft der Wissensehaften. 1900 . 
Körperchen, von welchen sie sich kaum unterscheiden lassen, wenn sie nicht geradezu von denselben rerdeckt werden.

Dem Pigmente kann man aus dem Wege gehen. Dasselbe ist ein Zellenbestandtheil, der erst in späteren Jahren auftritt, und lässt sich dadurch vermeiden, dass man zur Untersuchung Material von jugendlichen Individuen nimmt.

Schwerer sind die Nissl'schen Körperchen zu vermeiden. Wenn es auch zufällig gelingen könnte, zur Untersuchung solche Zellen zu erhalten, die im Stadium vollster Chromatolyse ständen, so ist doch darauf zu wenig zu rechnen. Um Resultate zu erhalten, müssen dieselben aus der Nervenzelle entfernt werden, und dieses gelingt in folgender Weise.

In Pikrinsäure-Sublimat gehärtete dünne Scheiben des Rückenmarkes werden vollständig von Pikrinsäure befreit, was in ziemlich kurzer Zeit zu erreichen ist, wenn dem 75 proc. Waschspiritus Lithium carbonicum zugesetzt wird. Darauf werden die Vorderhörner, wenn die Grösse des Rückenmarkquerschnittes dieses erlaubt, von der umgebenden weissen Substanz frei präparirt und mit ammoniakalischem Alkohol im Wärmeschrank behandelt. Die Zeit dieser Behandlung. variirt etwas und muss ausprobirt werden. Das Ammoniak wird nach beendigtem Aufenthalt im Brutschrank mit Salzsäure neutralisirt.

Schnitte von in dieser Weise behandelten Vorderhörnern lassen keine Tigroidfärbung mehr zu, andere Gewebe aber eine sehr deutliche Darstellung von Centrosomen. Es liegt also kein Grund zur Annahme vor, dass dieselben durch diese Behandlung an den Ganglienzellen ebenfalls ihr Vermögen, die Eisenhämatoxylinfärbung aufzunehmen, eingebüsst hätten oder gar gänzlich zerstört wären.

Eine andere Schwierigkeit liegt darin, dass diese Gebilde so klein sind, dass die Schnitte höchstens eine Dicke von $3 \mu$ haben dürfen, wenn man derselben habhaft werden will. Da die Vorderhornzellen aber bekanntlich recht gross sind, kann es leicht vorkommen, dass der Schnitt, welcher dieselben enthält, gerade verdirbt oder verloren geht. Um zum Ziele zu kommen, sind tadellose Serien der angegebenen Dicke absolute Bedingung, die aber bei guter Technik zu erfüllen ist.

Wie ich in meiner oben erwähnten Arbeit hervorgehoben habe, kann man nach der beschriebenen Vorbehandlung ebenfalls an älteren Rückenmarken trotz der Pigmentirung Resultate erhalten. Man muss nur den Gegenstand schon vorher aus Untersuchungen junger Zellen kennen und speciell die Entfärbung sicher beherrschen, dann gelingt es, Präparate zu erhalten, welche das Pigment in grauer, die Centrosomen aber in rein schwarzer Farbe zeigen. 
Wichtig ist noch, dass das Maternal möglichst frisch fixirt wird. An Rückenmarken, die später als 5 Stunden nach dem Tode in die Pikrinsäure-Sublimatlösung gebracht wurden, ist es mir nie gelungen, die Centrosomen zur Darstellung zu bringen.

Das Material, welches ich hier besprechen will, stammt von einem $37 \mathrm{~cm}$ langen Foetus, einem neugeborenen Kinde und einem ca. 40 Jahre alten Manne. Einige frühere fötale Stadien habe ich ebenfalls untersucht, dieselben waren aber etwas später nach dem Tode fixirt und gaben undeutlichere Präparate, so dass ich dieselben übergehe.

Am besten gelang hier die Darstellung der Centrosomen und der von denselben ausstrahlenden Radien an den Präparaten, welche vom Foetus stammten. Zufälligerweise erhielt ich denselben schon eine Stunde nach dem durch ein vorhergehendes Tranma bedingten Abort.

Diese Präparate zeigen alle bei genügender Entfärbung einen rein rothen Zellenleib, ebenso ist auch jede schwarze Chromatinfärbung aus den Kernen entfernt, nur der Nucleolus zeigt eine dunkelrothschwärzliche Färbung. Wenn die Zellenschnitte in der Serie nach erreichter derartiger Entfärbung sorgfältig Stück für Stück untersucht werden und zwar mit grösstmöglichster Vergrösserung und bester Beleuchtung, so trifft man stets einen Schnitt, in welchem sich eine eigenartige Structur bemerkbar macht. Dieselbe kennzejchnet sich oft durch eine hellere Färbung und erscheint alsdann von rundlicher Form. Vom Centrum dieses hellen Fleckens divergiren nach aussen feine Linien, deren Anzahl und Länge wechseln kann (Figg. 1 u. 2, Taf. II). Bisweilen liegen dieselben in vollkommen regelmässigen Abständen von einander (Fig. 2), ein anderes Mal begrenzen sie Keile verschiedener Grösse (Fig. 1).

Die Farbe dieser Strablen ist ein gesättigtes Roth, bedeutend dunkler als dasjenige des Protoplasmas. Sie scheinen aus aneinander gereihten Körnern aufgebaut zu sein, zwischen welchen eine etwas hellere, sie zusammenhaltende Substanz liegt.

Gerade im Centrum dieser strahlenförmigen Figur finden sich winzige schwarze Körner. Dieselben decken sich zuweilen theilweise, so dass es den Anschein hat, als ob hier nur ein einziges vorhanden wäre, welches dann aber keine glatten Umrisse und den einen Pol dunkler als den anderen zeigt.

Sehr oft, wohl entschieden in der Mehrzahl der Fälle, lassen sich zwei schwarze Körper unterscheiden, die neben einander liegen, aber durch einen hellen Zwischenraum deutlich von einander getrennt sind. An derartigen Präparaten ist es ohne allzugrosse Schwierigkeit möglich, einen oft recht beträchtlichen Unterschied in der Grösse festzustellen. 
Viel öfter aber sieht man den hellen Fleck noch mit einem Ringe aus ebenfalls dunkler gefärbten rothen Körnern umgeben. Dieser Ring lässt in seinem Aufbau nichts wahrnehmen, was ihn von den Strahlen unterscheiden könute. Derselbe schliesst in manchen Fällen die ganze Strahlenfigur $a b$, so dass ausserhalb desselben eine Fortsetzung derselben nicht wahrnehmbar ist. Stets ist dieses aber nicht die Regel, es kann gelingen, kurze Enden der Strahlen noch über den Ring hinaus zu verfolgen.

Bemerkenswerth ist weiter, dass der Durchmesser dieses Ringes in sehr grossen Breiten wechseln kann und zwar ohne dass ein Verhältniss zwischen Grösse der Zelle und der des Ringes besteht.

Was die Lage dieser ganzen hier zu beobachtenden Figur anbetrifft, so grenzt dieselbe nur selten an den Kern. Sie kann es thun, wie mehrere der beigegebenen Abbildungen zeigen, es kann aber auch ein Stück von demselben frei im Protoplasma liegen (z. B. Figg. 4 und 7).

Eine Einbuchtung des Kernes, welche dieser Sphäre entspräche und dieselbe aufnähme, wie es z. B. Studnička ${ }^{1}$ ) von den Gehirnnervenzellen bei Lophius darstellt, ist hier nicht aufzufinden.

Im Gegentheil zeigt der Kern hier sehr unregelmässige Umrisse, ist mit Einbuchtungen oder Vorsprüngen versehen, die in keiner Weise ein bestimmtes Verhältniss zu den beschriebenen Sphären aufweisen. Wie ich früher ${ }^{2}$ ) schon angeführt habe, steht das Vorkommen derselben auch hier nicht in Zusammenhang mit der Behandlung, welche die Nervenzellen durchgemacht haben, sondern es lassen dieselben sich ebenfalls an solchen Schnitten beobachten, die einer Ammoniakbehandlung nicht unterworfen gewesen sind.

An diesem Material war es die Regel, die Sphären in der grössten Protoplasmamasse, welche neben dem Kern in den Schnitt gefallen war, zu finden. Der Kern lag nämlich beinahe stets der einen Zellkante nahe gerückt.

Vollkommen so schöne Strahlenfiguren habe ich in den Vorderhornzellen des Neugeborenen nicht oft erhalten. Die Ursache liegt meiner Ansicht darin, dass mein Material nicht ebenso frühzeitig wie das vom Foetus fixirt werden konnte. Imomerhin habe ich eine genügende Anzahl von Präparaten gefunden, welche die Strahlen ebenso deutlich wie die eben beschriebenen Schnitte zeigen.

An den Figg. 9, 10 u. 11 sehen wir, dass hier ebenfalls der helle runde Fleck vorhanden ist. In seiner Lage stimmt derselbe ganz mit

1) Studnička, Beiträge zur Kenntniss der Ganglienzellen. 1. Ein neuer Befund von Centrosomen. Sitzber. d. kön. böhm. Gesellschaft d. Wissenschaften. Prag 1900.

2) Kolster, Anatomische Hefte. Heft 50. 
dem oben Hervorgehobenen überein. So liegt derselbe in Fig. 11 weit vom Kern entfernt, in den Figg. 9 u. 10 dem Kerne an.

Auch hier finden wir die gleichen, vom Centrum derselben entspringenden Strahlen, welche sich jedoch nur selten über den begrenzenden Ring hinaus zur Darstellung bringen lassen.

In der Mitte der Sphäre liegen ebenfalls die paarigen schwarzen Körper, manchmal sich deckend, ein anderes Mal deutlich getrennt neben einander.

Die Mehrzahl der Zellen bot allerdings ein etwas anderes Bild und zwar konnte man alle Uebergänge bei zu einem vollständigen Verwischtwerden der Strahlenfigur beobachten, ein meiner Ansicht nach ziemlich triftiger Grund, um die Fixirung für diesen Ausgang verantwortlich zu machen, und dieses um so mehr, als die Rückenmarksstücke, welche fixirt wurden, aus Versehen dicker genommen waren, als ich selber gethan hätte.

Recht hübsche Uebergangsformen zeigen z. B. die Figg. 12 u. 13. An denselben ist eine Andeutung der Strahlen noch sichtbar, obgleich dieselben nicht mehr so prägnant linienförmig erscheinen. Ebenso haben dieselben an ihrer Aufnahmefähigkeit für den rothen Farbstoff eine bedeutende Einbusse erlitten, wie auch die Zusammensetzung derselben aus einzelnen Körnern nicht mehr nachweisbar ist. Vollkommen heben sich nur die schwarzen Körperchen in der Mitte ab.

Ein etwas anderes Bild zeigen die Zellen, welche als Vorlage für die Figg. 14 u. 15 dienten. Hier jst von einer Strahlung überhaupt nicht mehr die Rede. Die schwarzen Centrosomen liegen innerhalb eines dunkler gefärbten, körnigen Hofes, der sich recht scharf von dem umgebenden Protoplasma durch seine abweichende Färbung abhebt.

Ob derartige Bilder aber allein der verspäteten Wirkung der Fixirflüssigkeit zugeschrieben werden dürfen, ist mir allerdings etwas zweifelhaft. Ich habe dieselben an anderem kunstgerecht eingelegtem Material ${ }^{1}$ ) allzuoft angetroffen, um nicht geneigt $\mathrm{zu}$ sein, hierbei Henneguy's ${ }^{2}$ ) Ansicht Raum geben zu wollen, nach welcher die verschiedene Darstellbarkeit der Sphären theilweise auf besonderen $\mathrm{Zu}$ ständen innerhalb der Zelle beruhen könnte.

In Fig. 16 ist dagegen das hier einfach erscheinende Centralkörperchen ohne jegliche charakteristische Umgebung und liegt mitten im Protoplasma. Sogar ein heller Hof lässt sich um dasselbe herum nicht auffinden.

1) Kolster, Ueber Centralgebilde in Vorderhornzellen der Wirbelthiere. Anatomische Hefte. Heft 50 .

2) Henneguy, Leçons sur la cellule. Paris 1896 . 
Aus den Präparaten, welche vom Rückenmark des ca. 40 Jahre alten Mannes stammen, will ich nur einige wenige Zellen abbilden und beschreiben. Dasselbe war recht spät in die Fixirungsflüssigkeit gebracht und bot, was die Protoplasmastructur anbelangt, nur geringe Andeutungen der an den oben beschriebenen Präparaten vorhandenen zierlichen Figuren. Wären nicht die schwarzen Körperchen durch ihre Farbintensität bei gewissen Entfärbungen so äusserst deutlich, so würde ich dieselben ganz bei Seite legen. Dieses um so mehr, als dieselben so gross sind, dass wir es hier sicher oft mit Verklumpungen oder Quellungen derselben zu thun haben. Dieses ist z. B. in Fig. 19 sicher der Fall, wie ich in folgender Weise feststellte.

Nachdem diese Figur gezeichnet war, wobei die Lage der Zelle vermittelst des Kreuztisches genau festgestellt war, entfernte ich den Balsam und ersetzte denselben nach den nöthigen Zwischenbehandlungen mit der Eisenoxyd-Ammoniaklösung. Nach Auflegen eines Deckglases, welches mit Paraffin an den 4 Ecken befestigt war, wurde die Zelle wieder eingestellt und speciell der schwarze Körper. Nach einiger Zeit, das Präparat wurde alle 10 Minuten untersucht, löste sich der schwarze Körper in zwei kleinere auf, welche mit denjenigen der Fig. 18 vollständig übereinstimmten, nur war ihre Farbe jetzt heller geworden und von derjenigen der herumliegenden Pigmentkörner nicht mehr zu unterscheiden. Dass zu dieser Verklumpungsfigur möglicherweise eine ungenügende Entfärbung der die Centralkörper verbindenden, ron Heidenhain als Centrodesmose bezeichneten Substanz beigetragen hat, ist sehr wahrscheinlich.

Sehen wir uns die beigegebenen Abbildungen, besonders die des Fötus und des Neugeborenen näher an, so ist es auffallend, in wie hohem Grade dieselben mit den Buehler'schen ${ }^{1}$ ) Abbildungen von den entsprechenden Gebilden in den Vorderhornzellen der Eidechse übereinstimmen. Der einzige Unterschied liegt darin, dass ich sein System von Linien an der Zellenoberfäche nicht habe auffinden können. Mein Material war für diesen Zweck wenig günstig, ebenso wie das früher von mir untersuchte, insofern, dass ich die Radien nie ein genügend langes Stück habe verfolgen können.

Von allen übrigen in Nervenzellen gefundenen weichen dieselben bedeutend ab. Schwer ist es wohl, auf Grund des bisher noch spärlichen Materiales zu sagen, worin der Grund liegt, ob wirklich so grosse Unterschiede zwischen Nervenzellen verschiedener Art vorhanden sind, oder ob dieselben Resultate der angewendeten verschiedenen Technik sind. Auch das eventuelle Fixiren in verschiedenen

1) Buehler, Protoplasmastructuren in Vorderhornzellen der Eidechse. Würzburger Verhandlungen, N. F. Bd. 29. 1895 . 
Functionsphasen muss beachtet werden, obgleich dasselbe nur für noch lebenswarm eingelegte Zellen von Bedeutung sein kann.

Während van Beneden ${ }^{1}$ ) diese Centralkörper gleich als permanente Zellorgane betrachtete, und in dieser Ansicht recht viel Anschluss fand, wurden auch im Laufe der Zeit ganz abweichende Ansichten geäussert.

In dieser Beziehung ist wohl in erster Hand die Schwierigkeit ihrer Nachweisung verantwortlich. Während es nämlich relativ schnell gelang, die Centralkörper bei den meisten in Theilungsphasen stehenden Zellen aufzufinden, stammen die sicheren Angaben über ihr Vorkommen auch in ruhenden Zellen erst aus späterer Zeit. In Folge dessen wollten mehrere Forscher, z. B. Hertwig ${ }^{2}$ ) geltend machen, dass die Centralkörper erst kurz vor der Theilung der Zellen entständen und dabei aus dem Kern hervorgingen.

Weiter hat Prenant ${ }^{3}$ ) wieder die Ansicht ausgesprochen, dass die Centralkörper das Resultat einer Ueberernährung der Zelle wären, welche Ueberernährung durch die Bildung derselben Anstoss zur Zelltheilung gebe.

Wie ich an anderem Orte $^{4}$ ) hervorgehoben habe, spricht aber das Auffinden der Centralkörper in Nervenzellen eines an perniciöser Anämie Verstorbenen doch für sich allein stark gegen die Annahme einer Ueberernährung als Ursache ihres Entstehens.

Die längere Zeit fehlenden Angaben über das Vorkommen der Centralgebilde in Nervenzellen suchte man sich in der Weise zu erklären, dass dieselben sich ja nicht mehr theilten. Direct auf ihr Vorkommen gerichtete Untersuchungen v. Lenhossek's ${ }^{5}$ ) liessen ihn zu dem Ausspruche kommen, dass bei Knochenfischen Andeutungen von Sphären in den centralen Nervenzellen vorhanden wären, dass dieselben sich aber bei erwachsenen Säugern nicht mehr nachweisen liessen. Dass v. Lenhossek bei diesem Ausspruch die möglichen technischen Schwierigkeiten, welche sich ihrer Darstellung hier entgegenstellen können, nicht berücksichtigte, ist wohl sicher.

Aus letzter Zeit haben wir aber auch einige Angaben zu ver-

1) Beneden et $\mathrm{Neyt}$, Nouvelles recherches sux la fécondation et la division mitosique chez l'ascaris megalocephal. Bull. etc. roy. Sc. de Belgique. 3. Série, T. XIV. 1887.

2) Hertwig, O., Die Zelle und die Gewebe. Jena 1892.

3) Prenant, Sur le corpuscule centrale. Bull. de la Société des Sciences de neurol. Série II. T. 18. 1894.

4) Kolster, Ueber Centralgebilde in Vorderhornzellen der Wirbelthiere. Anatomische Hefte. Heft 50.

5) v. Lenhossek, Ueber Nervenzellstructuren. Verh. d. anat. Gesellsch. Berlin 1896. 
zeichnen, nach welchen Theilungen der centralen Nervenzellen vorkommen könnten. $\mathrm{Ob}$ dieselben zu einer wirklichen Neubildung führen können, ist wohl noch sehr fraglich. Die Centralgebilde, ein bei der Karyokinese nach unseren jetzigen Kenntnissen derselben eine grosse Rolle spielendes Organ, sind aber, wie aus meinen Untersuchungen hervorgeht, in den centralen Nervenzellen sicher vorhanden, wodurch die Möglichkeit einer wirklichen Theilung dieser Zellen immerhin nicht ganz von der Hand gewiesen werden kann.

\section{Erklärung der Abbildnngen auf Tafel II.}

Die Figuren sind sämmtlich unter Anwendung von Zeiss apochromat. hom. Immersion $2 \mathrm{~mm}$ und Ocular 8 gezeichnet worden, nach Präparaten, welche die im Text angegebene Vorbehandlung durchgemacht haben und mit Bordeaux-Eisenhämatoxylin gefärbt waren.

Figg. 1-8 stellen Vorderhornzellen aus dem Rückenmark eines $37 \mathrm{~cm}$ langen menschlichen Foetus dar.

Figg. 9-16 sind Wiedergaben von Vorderhornzellen aus dem Rückenmark eines neugeborenen Kindes.

Figg. 17-18 entstammen Vorderhornzellen aus dem Rückenmark eines ca. 40 jährigen Mannes. 